\title{
Managing critically ill patients with severe traumatic brain injury: How should we season the recipe?
}

\author{
Paule Lessard Bonaventure, MD • François Lauzier, MD • Alexis F. Turgeon, MD
}

Received: 16 February 2016/Revised: 2 March 2016/Accepted: 17 March 2016/Published online: 4 April 2016

(c) Canadian Anesthesiologists' Society 2016

Maintaining adequate cerebral perfusion remains the overarching objective in the management of critically ill patients with severe traumatic brain injury. Despite limited evidence, the concept of maintaining normal intracranial pressure has been the driving force behind most interventions over the last few decades. The current guidelines for the management of severe traumatic brain injury advocate limiting brain edema using hyperosmolar therapy with mannitol to control elevated intracranial pressure. ${ }^{1}$ Nevertheless, concerns that the diuretic effect of mannitol induces hypovolemia have led to the popularity of using hypertonic saline solutions as an alternative optionthis despite limited evidence., ${ }^{2,3}$ Moreover, concerns that hyponatremia may lead to increased cerebral edema have led to a broader use of hypertonic saline as a continuous infusion with the goal of either normalizing or obtaining a greater than normal serum sodium. ${ }^{4}$

In this issue of the Journal, Griesdale et al. publish the results of a retrospective cohort study (two centres, $n=231$

P. L. Bonaventure, MD · F. Lauzier, MD

A. F. Turgeon, MD ( $\square)$

CHU de Québec - Université Laval Research Center, Population Health and Optimal Health Practices Research Unit,

Trauma - Emergency - Critical Care Medicine,

CHU de Québec - Université Laval, Québec City, QC, Canada

e-mail: alexis.turgeon@fmed.ulaval.ca

\section{P. L. Bonaventure, MD}

Division of Neurosurgery, Department of Neurological Sciences, CHU de Québec - Université Laval, Hôpital de l’Enfant-Jésus, Québec City, QC, Canada

F. Lauzier, MD · A. F. Turgeon, MD

Division of Critical Care Medicine, Department of

Anesthesiology and Critical Care Medicine,

CHU de Québec - Université Laval, Hôpital de l'Enfant-Jésus,

Québec City, QC, Canada patients) that aimed to evaluate the effect of a continuous $3 \%$ hypertonic saline infusion on the incidence of hypernatremia and mortality in patients with severe traumatic brain injury. ${ }^{5}$ This study provides new insights into this important topic in neurocritical care with findings that are not in agreement with the previous literature. The authors considered many relevant confounders in the adjusted analyses, including markers of trauma severity (abnormal pupillary reactivity, hypotension upon admission, and use of mannitol) and diabetes insipidus (use of desmopressin). Moreover, the multivariable stratified Cox regression analysis allowed consideration of the effect of time-since hypertonic saline solutions and hypernatremia were modelled as time-dependent variables. The investigators found no association between hospital mortality and continuous hypertonic saline infusion (hazard ratio [HR], 1.07; 95\% confidence interval [CI], 0.56 to $2.05 ; P=0.84$ ) or hypernatremia (HR, $1.31 ; 95 \%$ CI, 0.68 to $2.55 ; P=0.42$ ). Additionally, they observed that hypertonic saline did not modify the relationship between hypernatremia and death-i.e., among patients who received hypertonic saline, the occurrence of hypernatremia was not associated with increased mortality (HR, $1.52 ; 95 \% \mathrm{CI}, 0.16$ to $14.7 ; P=0.79$ ). Due to the nature of the intervention and the study design, the presence of residual confounding cannot be excluded, and the authors did not account for several other cointerventions. For example, patients who received hypertonic saline infusion seemed to have experienced a decrease in intracranial pressure, but other co-interventions that may impact on the control of intracranial pressure, such as sedatives, neuromuscular blockade, and therapeutic hypothermia (or controlled normothermia), were not measured. Also, the analysis of the impact of hypertonic saline infusion on the control of intracranial pressure was 
based on single daily values of intracranial pressure, thus greatly limiting the interpretability of results. Nevertheless, the results of this study contrast with some of the previously published literature. They raise several questions regarding the use of hypertonic saline solutions and the significance of hypernatremia in critically ill patients with severe traumatic brain injury.

\section{Does aiming for normal or greater than normal serum sodium levels improve outcomes in critically ill patients with severe traumatic brain injury?}

Conflicting clinical evidence and persistent concerns over the safety and efficacy of continuous infusion of hypertonic saline solutions have left some clinicians greatly questioning their use. Serum sodium disorders, particularly hypernatremia, are frequent in critically ill patients with traumatic brain injury and may arise from various mechanisms that may themselves impact differently on clinical outcomes. Hypernatremia resulting from water loss (e.g., diabetes insipidus) differs physiologically from iatrogenic sodium gain secondary to the use of hypertonic saline infusion. Tolerating or not treating hypernatremia due to water loss so as to avoid exacerbating cerebral edema may represent a totally different clinical scenario than iatrogenic hypernatremia from the administration of intravenous hypertonic saline solutions. Nevertheless, despite strong points of view from opinion leaders in the field, hypernatremia has never been associated with improved outcomes. It is particularly concerning that the results of a systematic review of cohort studies showed that hypernatremia was associated with serious complications such as renal failure, thrombotic events, and even mortality. ${ }^{6}$ This association was observed regardless of the etiology of the hypernatremia and mostly in the context of the use of hypertonic saline solutions. Also, a strong association between a very high sodium level and mortality was observed in a post hoc analysis of data from large-scale trials. ${ }^{7}$ Griesdale et al. did not evaluate a dose-gradient association mainly due to their limited sample size which precluded them from conducting such a subgroup analysis. Nevertheless, the association of adverse events with hypernatremia, which is seen with very high serum sodium values, may be reflective of the development of diabetes insipidus rather than the effect of hypernatremia per se, a potential confounder that Griesdale et al. controlled for in their study. It is thus unclear whether hypernatremia may be associated with increased mortality or whether it is simply a surrogate marker of the severity of the traumatic brain injury. Nonetheless, we certainly lack evidence to substantiate that targeting high serum sodium levels is beneficial in critically ill patients with traumatic brain injury.

\section{What is the physiologic rationale for using hypertonic saline solutions in the management of critically ill patients with severe traumatic brain injury?}

The use of hypertonic saline solutions was popularized as an alternative to mannitol as a hyperosmolar agent especially to avoid the potential complications associated with the latter. The classic teaching contends that raising serum sodium levels generates the osmotic gradient, which allows water to be drawn from the brain's interstitium and consequently lowers the intracranial pressure. ${ }^{8}$ This simple mechanism makes sense, but it also reassures us as to our ability to make a difference at the bedside based on theoretical physiological principles. Nevertheless, the clinical reality may be completely different, and what we observe in physiological studies may not apply in a critically ill individual. Indeed, as for any hyperosmolar agent, the integrity of the blood-brain barrier is a sine qua non condition to accomplish this theoretical targeted effect. It is well established that cerebral edema, e.g., from a brain contusion, and the integrity of the blood-brain barrier form a dynamic process in traumatic brain injury which not only varies from one cerebral area to another but also changes over time during the acute phase of care. Although acute bolus administration of a hyperosmolar solution was shown to be effective for the treatment of intermittent increased intracranial pressure by inducing a transient elevation of serum osmolarity, this effect may be transient and may even result in a subsequent paradoxical increase in intracranial pressure. It has been shown that this effect may be observed only in a non-contused brain, while contused brain areas may be more prone to increased edema following boluses of hypertonic saline. ${ }^{9}$ In a recent systematic review, we indeed observed no strong evidence for using hypertonic saline solutions vs mannitol for the management of increased intracranial pressure. ${ }^{10}$ In the absence of strong data to support the administration of hypertonic saline solutions, the use of this therapy as an infusion stems from inferences derived from consistent data showing an association between hyponatremia and unfavourable outcomes in this population. With repeated or continuous exposure to a hyperosmolar state, one can hypothesize that brain homeostasis will tend to reach a new equilibrium with increased content of intracellular electrolytes and an accumulation of idiogenic osmoles. This could lead to rebound cerebral edema and an elevation in intracranial pressure when the serum sodium normalizes. ${ }^{11}$ Our obsession to overnormalize and to overthink the 
homeostasis of critically ill patients with severe traumatic brain injury may lead us to use interventions that are not based on evidence and may even cause harm. ${ }^{12}$

This study by Griesdale et al. highlights the current lack of evidence surrounding the use of hypertonic saline infusion to sustain higher serum sodium levels in critically ill patients with severe traumatic brain injury. It does not support the use of hypertonic saline infusion in the management of critically ill patients with traumatic brain injury or the implementation of strict serum sodium targets. It certainly does not support targeting high serum sodium levels when caring for these patients. Rather, this study shows that achieving normal or greater than normal serum sodium values with hypertonic saline infusion, regardless of secondary hypernatremia, does not seem to impact mortality. Serum sodium may be just another marker of the severity of injury upon which no intervention can really have an effect. The only option that will help determine the exact role, if any, of hypertonic saline infusion in this specific population is conducting a large-scale randomizedcontrolled trial examining clinically significant functional outcomes.

\section{Gestion des patients en état critique avec traumatisme crâniocérébral grave. Comment devrions-nous adapter la recette?}

Le maintien d'une perfusion cérébrale adéquate reste l'objectif primordial de la gestion des patients en état critique à la suite d'un traumatisme crâniocérébral grave. En dépit de données probantes limitées, le concept du maintien d'une pression intracrânienne normale a été l'élément moteur de la majorité des interventions au cours des dernières décennies. Limiter l'œdème cérébral au moyen d'une thérapie hyperosmolaire sous forme de mannitol, est une méthode prônée par les lignes directrices actuelles pour la gestion des traumatismes crâniocérébraux graves afin de contrôler l'élévation de la pression intracrânienne. ${ }^{1}$ Cependant, des préoccupations concernant l'hypovolémie induite par l'effet diurétique du mannitol ont rendu populaire l'utilisation de solutions salines hypertoniques comme autre option thérapeutique en dépit de données probantes limitées. ${ }^{2,3}$ De plus, craignant que l'hyponatrémie cause une aggravation de l'œdème cérébral, les solutions salines hypertoniques en perfusion continue ont été plus largement utilisées dans le but de normaliser le taux sérique de sodium ou d'obtenir un taux de sodium sérique supérieur à la normale. ${ }^{4}$
Griesdale et coll. publient dans ce numéro du Journal, les résultats d'une étude de cohorte rétrospective (deux centres, $n=231$ patients) qui visait à évaluer l'effet d'une perfusion continue de solution saline hypertonique à $3 \%$ sur l'incidence de l'hypernatrémie et de la mortalité chez des patients victimes d'un traumatisme crâniocérébral grave. ${ }^{5}$ Cette étude apporte de nouveaux points de vue sur cet important sujet des soins neurologiques critiques avec des constations qui ne correspondent pas aux données de la littérature antérieure. De nombreux éléments confondants pertinents ont été envisagés dans les analyses ajustées, y compris les marqueurs de la sévérité $\mathrm{du}$ traumatisme (réactivité pupillaire anormale, hypotension à l'admission et utilisation de mannitol) et le diabète insipide (utilisation de desmopressine). De plus, l'analyse de régression multifactorielle stratifiée de Cox a permis d'envisager l'effet du temps (dans la mesure où les solutions salines hypertoniques et l'hypernatrémie ont été modélisées comme des variables dépendantes du temps). Les chercheurs n'ont trouvé aucune association entre la mortalité hospitalière et la perfusion continue de solution salée hypertonique (rapport de risque [HR]: 1,07; intervalle de confiance [IC] à $95 \%: 0,56$ à $2,05, P=$ $0,84)$ ou l'hypernatrémie (HR : 1,31 ; IC à $95 \%: 0,68$ à $2,55, P=0,42$ ). Ils ont également observé que la relation entre l'hypernatrémie et le décès n'était pas modifiée par la solution salée hypertonique; parmi les patients ayant reçu la solution salée hypertonique, la survenue d'une hypernatrémie n'a pas été associée à une augmentation de la mortalité (HR : 1,52; IC à $95 \%: 0,16$ à 14,$7 ; P=$ 0,79). Compte tenu de la nature de l'intervention et du plan de l'étude, la présence de facteurs confondants résiduels ne peut pas être exclue et plusieurs autres co-interventions n'ont pas été prises en compte. Par exemple, les patients qui avaient reçu une perfusion de solution salée hypertonique semblaient avoir eu une diminution de la pression intracrânienne, mais d'autres interventions pouvant avoir joué un rôle sur le contrôle de la pression intracrânienne comme les sédatifs, le bloc neuromusculaire et l'hypothermie thérapeutique (ou la normothermie contrôlée) n'ont pas été mesurées. L'analyse de l'impact des perfusions de solution saline hypertonique sur le contrôle de la pression intracrânienne a reposé sur des mesures quotidiennes uniques de cette pression, limitant ainsi considérablement les possibilités d'interprétation des résultats. Néanmoins les résultats de cette étude contrastent avec les articles publiés antérieurement et soulèvent la question concernant l'utilisation des solutions salées hypertoniques et la signification de l'hypernatrémie chez des patients en état critique avec un traumatisme crâniocérébral grave. 
L'évolution des patients en état critique présentant un traumatisme crâniocérébral grave est-elle améliorée par des interventions visant une natrémie normale ou supérieure à la normale?

Des données probantes contradictoires et des préoccupations persistantes concernant l'innocuité et l'efficacité des perfusions continues de solutions salines hypertoniques ont laissé certains cliniciens très perplexes quant à leur utilisation. Les troubles de la natrémie, en particulier l'hypernatrémie, sont fréquents chez les patients en état critique à la suite d'un traumatisme crâniocérébral grave et peuvent être dus à divers mécanismes ayant eux-mêmes un impact variable sur l'évolution clinique. L'hypernatrémie secondaire à une perte hydrique (par ex. diabète insipide) est physiologiquement différente d'un gain iatrogène de sodium secondaire à l'utilisation de perfusions de solution saline hypertonique. Tolérer ou ne pas traiter une hypernatrémie due à une perte hydrique pour éviter d'exacerber l'œdème cérébral peut représenter un scénario clinique totalement différent d'une hypernatrémie iatrogène due à l'administration de solutions salines hypertoniques. Néanmoins, en dépit de la conviction de chefs de file dans ce domaine, l'hypernatrémie n'a jamais été associée à une amélioration de l'état du patient. En revanche, il est préoccupant de constater dans une revue systématique des études de cohorte que l'hypernatrémie a été associée avec des complications graves, telles que l'insuffisance rénale, des événements thrombotiques et même des décès. ${ }^{6}$ Cette association a été constatée indépendamment de l'étiologie de l'hypernatrémie et essentiellement dans un contexte d'utilisation de solutions salines hypertoniques. Une forte association entre des taux de sodium élevés et la mortalité a également été observée dans une analyse a posteriori de données issues d'essais cliniques de grande envergure. ${ }^{7}$ Une telle association liée à la dose n'a pas été évaluée par Griesdale et coll. principalement en raison de la taille limitée de leur échantillon qui les a empêchés de mener une telle analyse de sous-groupe. Cependant, ces associations d'événements indésirables avec l'hypernatrémie observée avec des taux très élevés de sodium peuvent refléter l'évolution d'un diabète insipide plutôt qu'un effet de l'hyponatrémie proprement dite, un élément confondant qui a été contrôlé dans l'étude publiée dans ce numéro du Journal. On ne peut donc pas dire avec certitude si l'hypernatrémie peut être associée à une augmentation de la mortalité ou s'il s'agit simplement d'un marqueur accessoire de la grabité du traumatisme crâniocérébral. Quoi qu'il en soit, nous ne disposons avec certitude d'aucune donnée probante indiquant qu'il est bénéfique de viser des taux élevés de sodium sérique chez des patients en état critique à la suite d'un traumatisme crâniocérébral grave.
Quels éléments physiologiques justifie l'utilisation de solutions salines hypertoniques dans la gestion de patients en état critique à la suite d'un traumatisme crâniocérébral grave?

L'utilisation de solutions salines hypertoniques s'est généralisée comme alternative au mannitol en tant qu'agent hyperosmolaire, en particulier pour éviter les complications potentielles qui lui sont associées. L'enseignement classique nous apprend qu'en élevant les taux de sodium sérique, le gradient osmotique créé va permettre d'attirer l'eau hors du tissu interstitiel cérébral et, par conséquent, d'abaisser la pression intracrânienne. ${ }^{8} \mathrm{Ce}$ mécanisme simple a du sens, mais il nous rassure également sur notre capacité à faire une différence au chevet du patient à partir de principes physiologiques théoriques. Cependant, la réalité clinique peut être totalement différente et ce que nous observons dans les études physiologiques pourrait ne pas s'appliquer chez des patients dans un état grave. Comme pour tout agent hyperosmolaire, l'intégrité de la barrière hématoencéphalique est une condition sine qua non à l'obtention de l'effet théorique ciblé. Il est bien établi que l'œdème cérébral, comme dans le cas d'une contusion, et l'intégrité de la barrière hématoencéphalique forment un processus dynamique dans les lésions cérébrales traumatiques, variable selon la région du cerveau et aussi en fonction du temps au cours de la phase aiguë des soins. Bien qu'il ait été montré que l'administration aiguë, en bolus, d'une solution hyperosmolaire- induisant une élévation transitoire de l'osmolarité sérique-était efficace pour le traitement de l'augmentation intermittente de la pression intracrânienne, cet effet peut être seulement transitoire et même entraîner une élévation paradoxale ultérieure de la pression intracrânienne. Des auteurs ont montré que cet effet pourrait n'être observé que dans le tissu cérébral sans contusion alors que les régions cérébrales contuses auraient davantage tendance à une augmentation de l'œdème après des bolus de solution saline hypertonique. ${ }^{9}$ Nous avons effectivement observé, dans une revue systématique récente, qu'il n'existait pas de données probantes fortes en faveur de l'utilisation de solutions salines hypertoniques de préférence au mannitol pour la gestion de l'élévation de la pression intracrânienne. $^{10}$ En l'absence de solides données en faveur des solutions salines hypertoniques, l'utilisation de ce traitement sous forme de perfusions découle de données cohérentes montrant une association entre l'hyponatrémie et une évolution défavorable dans cette population. Avec une exposition répétée ou continue à un état hyperosmolaire, on peut supposer que l'homéostasie cérébrale tendra vers un nouvel équilibre avec une augmentation du contenu intracellulaire en électrolytes et 
une accumulation «d'osmoles idiogènes » qui pourraient entraîner un rebond de l'œdème cérébral et une élévation de la pression intracrânienne lorsque la natrémie se normalise. ${ }^{11}$ Notre obsession de surnormalisation et de surestimation de l'homéostasie chez les patients en état critique à la suite d'un traumatisme crâniocérébral grave peut nous conduire à utiliser des interventions qui ne sont pas basées sur des données probantes et qui pourraient même être nocives. ${ }^{12}$

Cette étude de Griesdale et coll. souligne l'absence actuelle de données probantes entourant l'utilisation de perfusions de solution salée hypertonique pour le maintien de taux élevés de sodium sérique chez des patients en état critique avec traumatisme crâniocérébral grave. Elle ne favorise pas l'utilisation de perfusion de solution saline hypertonique dans la gestion de patients en état critique à la suite d'un traumatisme crâniocérébral grave ou la visée de cibles strictes de sodium sérique. Elle ne soutient certainement pas la recherche de taux élevés de sodium sérique au cours des soins administrés à ces patients. Elle montre plutôt que l'obtention de concentrations sériques de sodium normales ou supérieures à la normale avec une perfusion de solution saline hypertonique ne semble pas avoir d'effet sur la mortalité, indépendamment de l'hypernatrémie secondaire. La natrémie pourrait n'être simplement qu'un autre marqueur de la gravité de la lésion sur laquelle aucune intervention ne peut véritablement agir. Seule la réalisation d'un essai clinique randomisé contrôlé de grande envergure étudiant les résultats fonctionnels cliniquement significatifs contribuera à déterminer le rôle exact éventuel de la perfusion de solution saline hypertonique dans cette population.

Conflicts of interest None declared.

Editorial responsibility This submission was handled by Dr. Philip M. Jones, Associate Editor, Canadian Journal of Anesthesia.

Conflits d'intérêts aucun déclaré.

Responsabilité éditoriale Cette soumission a été gérée par le Dr Philip M. Jones, Rédacteur adjoint, Journal canadien d'anesthésie.

\section{References}

1. Brain Trauma F, American Association of Neurological $S$, Congress of Neurological $S$, et al. Guidelines for the management of severe traumatic brain injury. II. Hyperosmolar therapy. J Neurotrauma 2007; 24 Suppl 1: S14-20.

2. Himmelseher S. Hypertonic saline solutions for treatment of intracranial hypertension. Curr Opin Anaesthesiol 2007; 20: 41426.

3. Berger Pelletier E, Emond M, Lauzier F, Savard M, Turgeon AF. Hyperosmolar therapy in severe traumatic brain injury: a survey of emergency physicians from a large Canadian province. PLoS One 2014; 9: e95778.

4. Ropper AH. Management of raised intracranial pressure and hyperosmolar therapy. Practical Neurology 2014; 14: 152-8.

5. Tan SK, Kolmodin L, Sekhon M, et al. The effect of continuous hypertonic saline infusion and hypernatremia on mortality in patients with severe traumatic brain injury: a retrospective cohort study. Can J Anesth 2016; 63: this issue. DOI: 10.1007/s12630016-0633-y.

6. Kolmodin L, Sekhon MS, Henderson WR, Turgeon AF, Griesdale $D E$. Hypernatremia in patients with severe traumatic brain injury: a systematic review. Ann Intensive Care 2013; 3: 35.

7. Van Beek JG, Mushkudiani NA, Steyerberg EW, et al. Prognostic value of admission laboratory parameters in traumatic brain injury: results from the IMPACT study. J Neurotrauma. 2007; 24: 315-28.

8. Ropper $A H$. Hyperosmolar therapy for raised intracranial pressure. N Engl J Med 2012; 367: 746-52.

9. Lescot T, Degos V, Zouaoui A, Preteux F, Coriat P, Puybasset L. Opposed effects of hypertonic saline on contusions and noncontused brain tissue in patients with severe traumatic brain injury. Critical Care Med 2006; 34: 3029-33.

10. Berger Pelletier E, Emond M, Lauzier F, Shields JF, Turgeon AF. Hypertonic saline in severe traumatic brain injury: a systematic review and meta-analysis of randomized controlled trials. CJEM 2016; DOI: 10.1017/cem.2016.12.

11. Gullans SR, Verbalis JG. Control of brain volume during hyperosmolar and hypoosmolar conditions. Annual Rev Med 1993; 44: 289-301.

12. Andrews PJ, Sinclair L, Rodriguez A, Eurotherm3235 Trial Collaborators, et al. Hypothermia for intracranial hypertension after traumatic brain injury. N Engl J Med 2015; 373: 2403-12. 